www.jmscr.igmpublication.org

Index Copernicus Value: 79.54

ISSN (e)-2347-176x ISSN (p) 2455-0450

crossrefDOI: https://dx.doi.org/10.18535/jmscr/v7i3.95

\title{
The Correlation between anti Mullerian Hormone with Progesterone / Estradiol Ratio on the day of HCG Administration in IVF Patients at Aster Fertility Clinic Hasan Sadikin Hospital Bandung
}

Authors

Arief Solehudin*, Mulyanusa Ritonga, Wiryawan Permadi, Tono Djuwantono

Department of Obgyn Hasan Sadikin Hospital, Aster Fertility Center Hasan Sadikin Hospital Bandung West Java Indonesia ${ }^{1,2}$

*Corresponding Author

Arief Solehudin

Contact no: +62-8112238250, Email: arief.ciaul@gmail.com

\begin{abstract}
Objective: To investigate the correlation between AMH with progesterone / estradiol ratio on the day of HCG administration in IVF patients.

Study design: Retrospective study including 43 women undergoing the GnRH antagonist protocol at the Aster Fertility Clinic Hasan Sadikin Hospital Bandung. Normal AMH is > $1.2 \mathrm{ng} / \mathrm{ml}$. Premature luteinisation is defined as progesteronelestradiol ratio $>1$ on the day of HCG administration and the sample is divided into premature lutenization if ratio $>1$ and control if ratio $<1$. Groups were correlated using Spearman.

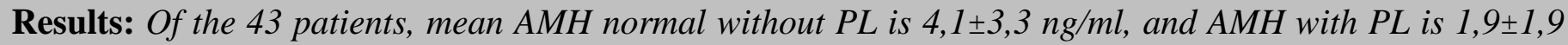
$\mathrm{ng} / \mathrm{ml}$. Control patients with $P$ / E2 <1 ratio were 39 patients (91\%), and patients with luteinized premature with a $P$ / E2 > 1 ratio were 4 (9\%). The mean age in patients with premature luteinization was $37 \pm 5$ years and control patients was $35 \pm 4$ years. In this study, there was a moderate, significant, and negative correlation between AMH with progesterone / estradiol ratio on the day of HCG administration (correlation coefficient $=-0,407$ with $p=0,007$ ).

Conclusion: The correlation between AMH with progesterone / estradiol ratio on the day of HCG administration in IVF patients is a moderate, significant, and negatif. Further research is needed regarding the function of cytokines and hormonal follicles that are reflected by AMH levels against premature luteinization
\end{abstract}

Keywords: premature luteinization, AMH, progesterone / estradiol ratio.

\section{Introduction}

Premature luteinization remains the most controversial topic in reproductive endocrinology and modern medicine. The pathogenesis and premature etiology of luteinization is debatable. ${ }^{1}$ Premature luteinization is usually defined as elevated serum progesterone levels prematurely at or before the day of hCG, which is thought to result from an earlier rise in preovulatory $\mathrm{LH}$. Most studies still use progesterone levels on hCG days as an early indication of luteinization, although cutoff rates differ between one study and 
another, ranging from 0.8 to $2 \mathrm{ng} / \mathrm{mL}$ (2.5-6.4 $\mathrm{nmol} / \mathrm{L}){ }^{1,2,3}$

Some researchers have recently shown that there is a difference between premature luteinization that develops in the natural cycle and that occurs during controlled ovarian hyperstimulation. The pathogenesis of premature luteinization in non$\mathrm{GnRH}$ analog cycles is believed to result from an increase in preovulatory LH levels. However, the etiology of early luteinization in this cycle is still under investigation. Most studies agree that premature luteinization in the non-GnRH analog cycle is associated with poor maturation, recovery, fertilization, and poor reproductive quality and is associated with low rates of pregnancy and high abortion. ${ }^{1,2}$

This study proved another definition of premature luteinization, taking into account the "physiological" increase in the final follicular phase in women undergoing controlled ovarian hyper stimulation. Premature luteinization is defined as the P/E2> 1 ratio on the day of hCG administration. Prior research, premature luteinization is associated with low ovarian reserve, in women with unexplained infertility who undergo controlled ovarian hyper stimulation with hMG. ${ }^{1,2}$ If premature luteinization shows low ovarian reserve in non GnRH agonist cycles, then this may also occur in the GnRH agonist cycle. $^{1,2,3,4,5,6}$.

High $\mathrm{P}$ levels occur with an increase in the number of follicles and levels of estradiol (E2) is high. Thus, the increase in progesterone in the final follicular phase reflects the total amount of progesterone secreted by adult cohort follicles. This result has resulted in the idea that the P/E2 ratio can differentiate the secreted $\mathrm{P}$ from the mature follicle of $\mathrm{P}$ removed from multiple follicle follicles; it has been suggested that the $\mathrm{P} / \mathrm{E} 2$ ratio reflects both premature luteinization and clinical outcomes for IVF and ICSI-ET cycles more accurately than $\mathrm{P}$ levels alone. ${ }^{3,4,5,6}$

Several studies, however, have evaluated the effect of the P/E2 ratio on IVF and ICSI-ET outcomes, and different cut-off values. All studies used a long agonist protocol. Some of these studies used the P/E2> 1 ratio and suggested that it was associated with poor pregnancy outcome and poor ovarian response. ${ }^{3,5}$

In women, anti-Mullerian hormone (AMH) levels may represent the ovarian follicular pool and could be a useful marker of ovarian reserve. The clinical application of AMH measurement has been proposed in the prediction of quantitative and qualitative aspects in assisted reproductive technologies (ART). In women AMH is produced by granulosa cells, from pre-antral and antral follicles and the main physiological role of AMH in the ovary seems to be limited to the inhibition of the early stages of follicular development. $\mathrm{AMH}$ is produced by granulosa cells from preantral and antral follicles, restricting expression to growing follicles, until they have reached the size and differentiation state at which they are selected for dominance by the action of pituitary FSH. In the human this occurs in antral follicles of size 4$6 \mathrm{~mm}^{7}$

Current theories also suggest a role for $\mathrm{AMH}$ as a co-regulator of steroidogenesis in granulosa cells, as $\mathrm{AMH}$ levels appear to be related to estradiol levels in follicular fluid from small antral follicles.

This is confirmed by a recent study which showed that polymorphisms in the gene for AMH or AMH receptor type II seem to be related to follicular phase estradiol levels, suggesting a role for $\mathrm{AMH}$ in the $\mathrm{FSH}$-induced steroidogenesis in the human ovary. ${ }^{7}$

AMH levels seem to decline gradually during gonadotrophin administration as a part of controlled ovarian stimulation (COS). The reduction of $\mathrm{AMH}$ levels during $\mathrm{COS}$ could be due to a negative direct or indirect effect of FSH on ovarian AMH secretion. During exogenous administration of FSH there is an increase in estradiol levels, which could be a reason for decreased AMH. Indeed estradiol has been implicated in the down-regulation of $\mathrm{AMH}$ and AMHII mRNA in the ovary. Stimulation with FSH induces growth of follicles that enlarge and lose their AMH expression, and this is probably 
the main reason for $\mathrm{AMH}$ reduction. Hence, due to the reduction of $\mathrm{AMH}$ levels during $\mathrm{FSH}$ administration, AMH measurement to predict the ovarian response to FSH should not be performed during gonadotrophin treatment, but some months to some days prior commencing FSH treatment. Much data show a strong and positive correlation between basal AMH serum levels and the number of retrieved oocytes in women undergoing ovarian stimulation. $^{7}$

Fran chin investigate the possible influence of follicular maturation and luteinization on antimüllerian hormone (AMH) secretion and the relationship between per-follicle AMH levels, ovarian follicular status, and responsiveness to controlled ovarian hyper stimulation $(\mathrm{COH})$. Both final follicular maturation and luteinization interfere with granulosa cell AMH production. The relationship between intrafollicular AMH content, the surrounding follicular status, and ovarian response to $\mathrm{COH}$ indicates that peripheral AMH levels reflect not only follicle count but also per-follicle AMH production. ${ }^{8}$

AMH levels in follicular fluid were found to be roughly three times higher in small than in large follicles confirming the hypothesis that AMH production by granulosa cells probably declines during final follicular maturation. Moreover in both small and large follicles, follicular fluid AMH levels correlated positively eighth the number of early antral follicles on cycle day 3 before COS, growing follicles on the day of hCG administration and oocytes retrieved. This interesting finding may indicate that peripheral AMH levels are not exclusively dependent on the number of follicles; they are also modulated by individual follicular ability to produce AMH. Hence, elevated peripheral AMH levels indicate not only that the number of antral follicles is increased, but also that each follicle probably produces more AMH individually. This offers us a new understanding of the reported association between peripheral AMH levels and the ovarian fertility potential, and leads the authors to speculate that serum AMH measurement could reflect not only quantitative but also qualitative ovarian responsiveness to $\mathrm{COS}{ }^{7}$

In adult women, the possible effects of granulosa cell luteinization on AMH production are less well documented. Previous data indicate that isolated corpora lutea from rats express minimal amounts of $\mathrm{AMH}$ and its type II receptor mRNA as compared with small and large antral follicles. Moreover, in a cohort of women undergoing $\mathrm{COH}$ for in vitro fertilization and embryo transfer (IVFET), AMH levels in pooled follicular fluid remained detectable 34 hours after human chorionic gonadotropin (hCG) administration. Conclusive clinical data on the possible influence of the degree of follicle luteinization on AMH production is currently not available.

Besides these uncertainties concerning the influence of follicular development and luteinization on $\mathrm{AMH}$ production, some clinical studies have reported a quantitative relationship between peripheral AMH levels and the number of early antral follicles, the reliability of which sur- passes conventional hormonal biomarkers of ovarian follicular status. However, it still remains unclear whether increased peripheral AMH levels reflect exclusively the number of early antral follicles or are also due to increased per-follicle $\mathrm{AMH}$ secretion. Insights into this sensitive issue could help to clarify the role of AMH not only as a quantitative but also as a qualitative indicator of ovarian follicular status.

Hence, the present investigation analyzed serum and follicular fluid samples obtained from IVF-ET candidates' follicles at two different development stages to assess the possible modulating role of follicular maturation and luteinization on AMH secretion, and the relationship of perfollicle AMH levels, ovarian follicular status, and responsiveness to $\mathrm{COH}^{8}$

The purpose of this study was to to investigate the correlation between $\mathrm{AMH}$ with progesterone / estradiol ratio on the day of HCG administration in IVF patients GnRH antagonist cycles. 


\section{Methods}

Population as subject in this research is female patient who follow IVF program at Aster Hospital Clinic Hasan Sadikin Hospital Bandung in 2017. This research data was obtained from medical record of RSHS Aster clinic patients who meet the criteria of this study. Place at ASTER RSHS Bandung clinic. The study time was between August and October 2018. The analysis was performed using SPSS 24.0 program.

The method of this study is a retrospective study conducted on RSHS Aster infertility clinic patients undergoing therapy in 2017. In this study data analysis of 85 patients undergoing IVF but we found 43 cycles of patients undergoing TRB program fulfilled the criteria.

The P/E2ratio was calculated as $\mathrm{P}(\mathrm{ng} / \mathrm{mL})$ $1000 / \mathrm{E} 2(\mathrm{pg} / \mathrm{mL})$. The primary outcome measure was ongoing pregnancy, defined as pregnancy continuing beyond the live birth. Normal AMH level was $1.2 \mathrm{ng} / \mathrm{ml}$.

\section{Results}

Of the 43 patients, mean AMH normal without PL is $4,1 \pm 3,3 \mathrm{ng} / \mathrm{ml}$, and $\mathrm{AMH}<1,2 \mathrm{ng} / \mathrm{ml}$ with PL is $1,9 \pm 1,9 \mathrm{ng} / \mathrm{ml}$. Control patients with $\mathrm{P} / \mathrm{E} 2<1$ ratio were 39 patients $(91 \%)$, and patients with luteinized premature with a $\mathrm{P}$ / E2> 1 ratio were 4 $(9 \%)$. The mean age in patients with premature luteinization was $37 \pm 5$ years and control patients was $35 \pm 4$ years. In this study, there was a moderate, significant, and positif correlation between AMH with progesterone / estradiol ratio on the day of HCG administration (- 0,407 with $\mathrm{p}$ $=0,007)$.

Table 1 Correlation between AMH with P/E2 Ratio

\begin{tabular}{lcc}
\hline & $\mathrm{r}$ & $\mathrm{p}$ \\
\hline AMH & $-0,407$ & 0,007 \\
P/E2 & &
\end{tabular}

Note: Premature luteinisation is defined as progesterone/estrogen ratio $>1$ on the day of HCG administration and the sample is divided into premature lutenization if ratio $>1$ and control if ratio $<1$. (significant if $p$ value $<0,05$ )

\section{Discussion}

This offers us a new understanding of the reported association between peripheral AMH levels and the ovarian fertility potential, and leads us to speculate that serum AMH measurements could reflect not only quantitative but also qualitative ovarian responsiveness to $\mathrm{COH}$. The present study was designed to examine, in individual follicles, the hypothesis that the degree of follicular maturation and luteinization influences $\mathrm{AMH}$ production. Also, it aimed at clarifying whether the reported quantitative relationship between peripheral AMH levels and the number of early antral follicles might be affected by their individual ability to produce AMH. For achieving these objectives, $\mathrm{COH}$ for IVF-ET represented a unique model to quantify not only the antral follicle responsiveness to exogenous FSH but also the $\mathrm{AMH}$ production in large and small follicles in the same patient.

$\mathrm{AMH}$ levels are roughly three times as high in small as in large follicles. These data are consistent with the hypothesis that AMH production by granulosa cells probably declines during final follicular maturation, providing direct confirmation to our previous findings that showed a progressive decline in serum AMH levels during the evolution of ovarian follicles from the early antral stage to the preovulatory stage during $\mathrm{COH}$. Both the physiologic mechanisms implicated in the reduction of $\mathrm{AMH}$ production by maturing follicles and the possible consequences of this phenomenon on the regulation of folliculogenesis remain unclear. It is possible that the increase in granulosa cell sensitivity to FSH that occurs during the ultimate stage of folliculogenesis and the down regulation of $\mathrm{AMH}$ and its type II receptor mRNA are interrelated phenomena. Further studies are needed to clarify these issues and to provide insights into the role of $\mathrm{AMH}$ during the final follicular maturation. Our observation that $\mathrm{P} 4$ levels were lower in small follicles compared with large follicles may be explained by the fact that the expression of $\mathrm{LH} / \mathrm{hCG}$ receptors in granulosa cells probably is 
less intense in small follicles compared with large follicles. Also, the similar follicular fluid $\mathrm{E}_{2}$ levels between small and large follicles may be due to the potentially different luteinization status of the two follicular classes. Assuming that follicular luteinization probably is milder in smaller than larger follicles, the transient decrease in $\mathrm{E}_{2}$ production that accompanies the luteinization process possibly was attenuated in small follicles, too. The negative relationship between follicular fluid AMH and progesterone levels observed in both follicular classes supports the hypothesis that follicle luteinization exerts a negative effect on the production of AMH by granulosa cells. Baarends et al. have previously demonstrated that $\mathrm{AMH}$ and its type II receptor mRNA expression are markedly reduced in the isolated corpora lutea of rats, compared with small and large antral follicles. Expanding this observation, our present results suggest that the more advanced the process of luteinization of granulosa cells, as reflected by the magnitude of intrafollicular progesterone secretion, the lesser their ability to secrete AMH. If confirmed, these results may also constitute an alternative explanation to the overall increased AMH levels observed in small follicles.

Accordingly, the positive correlation between serum and follicular fluid AMH levels clearly indicates that peripheral AMH levels are not exclusively dependent on the number of follicles; they also are modulated by their individual ability to produce $\mathrm{AMH}$. Hence, elevated peripheral AMH levels indicate not only that the number of antral follicles is in- creased, but also that each follicle probably produces more AMH individually. This offers us a new understanding of the reported association between peripheral AMH levels and the ovarian fertility potential, and leads us to speculate that serum AMH measurements could reflect not only quantitative but also qualitative ovarian responsiveness to $\mathrm{COH}^{8}$

The detrimental effect of high estradiol level on uterine receptivity has been discussed for many years. Other study has suggested that high responders (estradiol on $\mathrm{hCG}$ day $>3,000 \mathrm{pg} / \mathrm{mL}$ ) have significantly lower implantation and pregnancy rates and impaired endometrial receptivity. However this study failed to find a detrimental effect of high estradiol level on hCG day on pregnancy outcome. Other studies proposed that not only estradiol level, but also $\mathrm{P}$ level on hCG day affects pregnancy outcome. However, the unfavourable effect of elevated $\mathrm{P}$ on pregnancy outcome was questioned by yet other studies. ${ }^{1,2}$

In the late follicular phase of $\mathrm{COH}, \mathrm{P}$ level reflects the total amount of $\mathrm{P}$ secreted by maturing follicles. The P levels have been found to correlate positively with the number of mature follicles and with estradiol levels on the day of hCG administration. Thus, using a single hormone level to predict pregnancy outcome is confounding and the influence of both estradiol and $\mathrm{P}$ should be taken into consideration. ${ }^{1,2}$

In the present study, the roles of $\mathrm{P}$ and the $\mathrm{P} / \mathrm{E} 2$ ratio in high responders were explored. The data showed that women with higher P levels had higher E levels. This result is in agreement with the positive correlation between $\mathrm{P}$ and E2in the late follicular phase. Considering the secretion of $\mathrm{P}$ and E2 from granulosa cells in late follicular phase and the interaction of both $\mathrm{P}$ and E2on endometrium. An elevation of P/E2ratio on hCG day be used as definition of premature luteinization. Younis et al. implied that defining premature luteinization as $\mathrm{P} / \mathrm{E} 2>1$ could differentiate physiologic $\mathrm{P}$ secretion from multiple healthy mature follicles from that secreted from dysmature follicles; the latter could be related to low ovarian reserves and poor pregnancy outcome. $^{1,2}$

The clinical application of AMH measurement has been proposed in the prediction of quantitative and qualitative aspects in assisted reproductive technologies (ART). It is extensively recognized that pregnancy in ART is mostly related to the qualitative than quantitative aspects of IVF. As the status of the ovarian reserve includes both the 
quantity and quality of ovarian fol- licle pool, AMH may reflect not only quantitative but also qualitative ovarian responsiveness. In order clarify the complex relationship between $\mathrm{AMH}$ and oocyte quality, embryo quality and implantation and pregnancy rate, we should separately comment on studies of AMH in the follicular fluid and in serum.

Our study shows that the higher the AMH value, the smaller the occurrence of luteinization premature. Normal AMH values not only assess follicle quantity, but can also access follicular quality. Low follicles with $\mathrm{AMH}$ will produce progesterone and estrogen with an abnormal ratio, so that when injecting $b$ HCG, there is an abnormal ratio of progesterone and estrogen. This may be due to the condition of the follicles that are dismissed, so the steroid hormone produced is not good. So that the higher the AMH value, the less likely the occurrence of premature lutenization, then AMH basal examination can be used to predict the occurrence of premature luteinization.

\section{Conclusion}

The correlation between AMH with progesterone / estradiol ratio on the day of $\mathrm{HCG}$ administration in IVF patients is a moderate, significant, and positif. Further research is needed regarding the function of cytokines and hormonal follicles that are reflected by AMH levels against premature luteinization

\section{Acknowledgement: None}

\section{References}

1. Johnny S. Younis,Moshe Matilsky,Orit Radin,and Moshe Ben-Ami.Increased progesterone/estradiol ratio in the late follicular phase could be related to low ovarian reserve in in vitro fertilizationembryo transfer cycles with a long gonadotropin-releasing hormone agonist.Fertility and Sterility 2001; 76: 2 .
2. Fa-Kung Lee, Tsung-Hsuan Lai, Tseng-Kai Lin, Shang-Gwo Horng, and Su-Chee Chen. Relationship of progesterone/ estradiol ratio on day of hCG administration and pregnancy outcomes in high responders undergoing in vitro fertilization. Fertility and Sterility 2009;92:4

3. Esra S. Cetinkaya, Bulent Berker, Rusen Aytac, Cem Atabekoglu, Murat Sonmezer, Batuhan Ozmen. The value of the progesterone-to-estradiol ratio on the day of hCG administration in predicting ongoing pregnancy and live birth rates in normoresponders undergoing GnRH antagonist cycles.European Journal of Obstetrics \& Gynecology and Reproductive Biology 2013;452-457.

4. Aboubakr M. Progesterone rise on the day of HCG administration (premature luteinization) in IVF: An overdue update. J Assist Reprod Genet 2010;27:149-155.

5. Melo M, Meseguer M, Garrido N, Bosch E, Pellicer A, Remohi J. The significance of premature luteinization in an oocytedonation programme.Human Reproduction 2006;21.

6. Wiweko B, Natadisastra M, Soebijanto S, Sumapraja K, Hestiantoro Effect of premature luteinization on IVF outcome. A Faculty Of Medicine University Of Indonesia. Reproductive BioMedicine Online 2008; 16.

7. Marca A, Sighinofil G, Argento C, Baraldi E. Anti-Mullerian hormone (AMH) as a predictive marker in assisted reproductive technology (ART). Human Reproduction Update, Vol.16, No.2 pp. 113-130, 2010

8. Fanchin R, Louafi N, Lozano M. Perfollicle measurements indicate that anti-müllerian hormone secretion is modulated by the extent of follicular development and luteinization and may reflect qualitatively the ovarian follicular status. Fertil Steril. 2005 Jul;84(1):167-73). 